\title{
Iterative Learning Synchronized Control of Multi-leaf Collimator Based on Cross-coupled Control
}

\author{
Xin Zhang ${ }^{*}$, Wenru Lu, Zhongcui Miao, Ziyun Jiang, Wenbo Xu
}

School of Automation \& Electrical Engineering, Lanzhou Jiaotong University, Lanzhou 730070, China

Corresponding Author Email: zhangx@mail.lzjtu.cn

https://doi.org/10.18280/ejee.210509

Received: 1 May 2019

Accepted: 12 August 2019

\section{Keywords:}

iterative learning control, synchronized control, cross-coupled control, multileaf collimator, conformal radiotherapy

\begin{abstract}
The purpose of this research was to ensure that the leaves of multi-leaf collimator (MLC) move along the trajectory set in the radiotherapy plan. Aiming at the characteristics of multileaf control of multi-leaf collimator, the model of leaf control system is established. Taking the synchronized error between leaves as the input of the system, combined with PD CCC and PD ILC, an iterative learning control strategy based on cross-coupled is proposed, and verified by experiments, then compared with the multi-leaf control strategy that adopts iterative learning control algorithm independently. The experimental results show that the control strategy proposed in this study can reduce the synchronized error between leaves, and the initial value of the maximum absolute value of the error proposed reduces the synchronous position error of the leaves from the beginning, which meets the requirements of radiation therapy. The results provide a guarantee for the synchronous control of MLC to improve the efficiency of conformal radiotherapy.
\end{abstract}

\section{INTRODUCTION}

With the further development of radiotherapy technology, the research on the improvement of radiotherapy equipment has also been greatly developed. In the course of radiotherapy, the precise location and the dose correction of the tumor target are of critical importance [1]. The main purpose of multi-leaf collimator (MLC) is to achieve conformal radiotherapy, which accurately illuminates the lesion target [2]. The radiotherapy system addresses the following main problems: medical image segmentation, medical volume data structure based on body tissue contour, three-dimensional surface reconstruction algorithm based on medical volume data, three-dimensional dose calculation model, and the control system of MLC [3]. The main function of the MLC control system is to control the leaves to the specified position accurately, improve the dose accuracy at each point within the control irradiation field, reduce the complications of surrounding normal tissue, and effectively improve the gain ratio of treatment in the radiation therapy. Therefore, during the radiotherapy process, the movement of each leaf of MLC and the position accuracy of the leaves need to be controlled precisely. To control the MLC regardless of which method adopted, three elements must be considered: the leaf drive unit, the real-time monitoring of leaf position, and the control logic and strategy of leaf movement. The leaf drive unit of electric MLC includes two types of motors, namely digital or analog ones. The measure of monitoring leaf position involves the use of mechanical limit switches, optical imaging systems, and linear encoders. The control logic and strategy of leaf involves the determination of leaf speed and the leaf position in consideration of the dose compensation. The multi-leaf collimator consists of a plurality of leaves, each of which is driven by a motor. It is a multi-motor motion system. The control accuracy of MLC depends on the control accuracy of a single motor and the Synchronized position error of multiple motor.

Much research has been done on the position control accuracy of a single leaf of the MLC, Zaferullah designed a double closed-loop controller of an MLC leaf drive unit based on the field-programmable gate array (FPGA) [4]. However, the scholar did not mention the correspondence between controller and closed-loop system. Xin tuned the parameters of the PID controller by the weighted particle swarm optimization (PSO) algorithm, such that the MLC leaf accuracy satisfies radiotherapy requirements [5]. Nevertheless, the goodness of fitness of the weighted PSO algorithm is subpar according to the experimental results. The method becomes premature and fails to converge to the global optimal solution. What is worse, the windup phenomenon occurs due to the presence of the integral part [6]. To overcome these defects, Xin designed a fractional anti-windup controller after considering the collision-induced position error of the leaves. The new controller can keep the leaves exactly in the specified positions in no time, enable the system to resume the stable operation state, and enhance the anti-interference ability of the system [7].

In fact, the MLC control system has to tackle the synchronized motion of multiple motors [8]. Aside from the accuracy of single-leaf position, the influence of the position error of adjacent leaves should also be taken into account. In traditional multi-motor motion control systems [9], the control strategy largely depends on single motor control, such as PID control, sliding mode control, and iterative learning control. The problem is the tracking performance of a single motor cannot guarantee the control of synchronized position error in the multi-motor system. The control effect will not be desirable if the associated motors are poorly synchronized in motion. The synchronized position error index must be emphasized over single motor position error index, so as to achieve desirable synchronized control performance of multi- 
motor system.

Some of the representative works on the synchronized position error of multiple motors are reviewed below. Since its proposal by Koren [10], the cross-coupled control strategy has been extensively applied to the research on multi-motor or multi-axis motion control system. For instance, Dong et al. [11] from City University of Hong Kong explored the formation control of multiple mobile robots following the theory of cross-coupled synchronized control. Lan [12] from Harbin Institute of Technology implemented cross-coupled control theory in the synchronized position control of fingers and single-finger synchronized impedance control. Zhong et al. [13] put forward a strategy to realize accurate synchronized position control of multi-axis servo motion.

In the whole process of dynamic intensity-modulated radiation therapy, the motion of the leaf of MLC is a kind of repetitive movement process. Iterative learning control is applied to the intermittent repetitive motion, which pursues the complete tracking in a limited time [14-15]. In this paper, an iterative learning control strategy based on cross-coupling is proposed, which satisfied the control requirements of leaf repetitive motion while considering the synchronized error between leaves.

To sum up, the above theoretical and empirical studies have laid a good foundation to the precision research and synchronized control of multiple leaves of the MLC.

The remainder of this paper is organized as follows: Section 2 introduces the working principle of the MLC, On the basis of establishing the motion system model of MLC, iterative learning synchronized control strategy based on cross-coupled are proposed for dynamic IMRT. Section 3 carried out experiments to verify the performance of the proposed control strategy. Section 4 wraps up this paper with some meaningful conclusions.

\section{MATERIALS AND METHODS}

\subsection{Working principle multi-leaf collimator}

Multi-leaf collimator is composed of electronic control circuit, mechanical drive device and leaves which are arranged in pairs. In the radiation therapy system, the positions of the leaves of MLC are determined based on the shape of the tumor. The shape of the rays that pass through the MLC is similar to the projection on the irradiated surface of the tumor. The diagram of the working principle of MLC is shown in Figure 1.

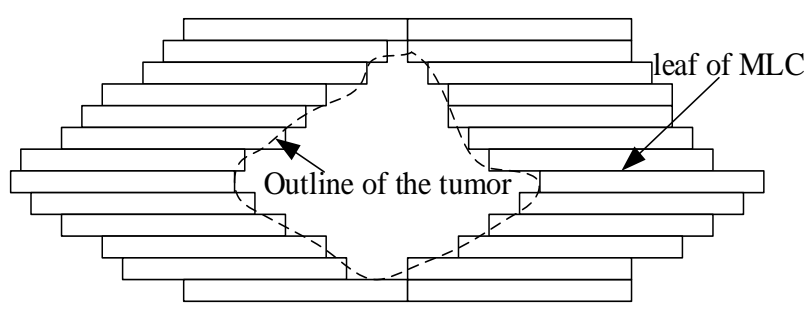

Figure 1. Working principle diagram of multi-leaf collimator

In practical applications, each leaf is driven by a microelectric motor, and the rotational movement of the motor is converted to a linear motion of the leaf through a screw rod. Each leaf has a set of independent controller. The electric motor used in this system includes stepping motor, DC servomotor, and brushless DC motor. To ensure the accuracy of the leaves in place, the following methods are used: (1) accurately control the speed, acceleration, number of revolutions or steps of the drive motor to achieve control accuracy of the leaf in position (this requires the use of encoders to detect the operating status of the motor); and (2) direct detection of the position of the leaves [16] and identification the next running requirements of the motor (commonly used methods in two-dimensional ionization chamber matrix to verify the precision of the leaves position of MLC). The BLDC motor can be controlled through the drive file of MLC that is generated by the radiation treatment planning system and contains the location information for each leaf. In the course of static intensity modulated radiation therapy, each irradiation field is divided into several sub fields by means of MLC. In the course of the treatment, the leaves stop after moving to the prescribed position of the first sub-field, the accelerator turns on the rays and turns off the rays after reaching the specified dose. Subsequently, the leaves move to the next sub field, until the end of treatment, the ray intensity of all subfields is added to form the required intensity distribution. Each sub-field corresponds to the positions of the leaves of MLC; therefore, it is necessary to control the leaves of MLC precisely to achieve the purpose of radiation therapy.

\subsection{ILC synchronized control strategy of leaves of MLC based on cross-coupled}

A model of a multi-motor motion control system [17-18] can be expressed as Eq. 1:

$$
M(x) \ddot{x}+C(x, \dot{x}) \dot{x}+F(x, \dot{x})=\tau
$$

where, $\tau$ is the $\mathrm{n} \times 1$ control input vector, $x$ is the $\mathrm{n} \times 1$ coordinate vector, $M$ is the positive definite inertial matrix, $C$ is the Coriolis force vector, $F$ is the $\mathrm{n} \times 1$ friction disturbance vector.

Definition 1: single leaf tracking error (position error) is:

$$
e=x^{d}-x
$$

where, $x^{d}$ is a constant given value, $x$ is the real-time acquisition value.

\subsubsection{Synchronized control strategy}

In this paper, the balanced control strategy is adopted. The control strategy means that the controlled system does not have a unified master leaf. Each leaf is a master leaf and a slave leaf. Each leaf is synchronized with each other. In each control cycle, each leaf receives the actual position value of the other leaf as a reference for its own position reference via the control network.

In the control system of MLC, the Synchronized requirements for each leaf may not be exactly the same. To simplify the design, it is assumed that the synchronized requirements for each leaf are the same, as described in Eq. 3:

$$
e_{1}=e_{2}=\cdots=e_{n}
$$

Definition 2: The Synchronized error is the difference between the tracking error of the current leaf and the adjacent 
leaf, as shown in Eq. 4.

$$
\begin{aligned}
& \varepsilon_{1}=e_{1}-e_{2} \\
& \varepsilon_{2}=e_{2}-e_{3} \\
& \vdots \\
& \varepsilon_{i}=e_{i}-e_{i+1} \\
& \vdots \\
& \varepsilon_{n-1}=e_{n-1}-e_{n} \\
& \varepsilon_{n}=e_{n}-e_{1}
\end{aligned}
$$

where, $\varepsilon_{i}$ is the Synchronized error of $i$ th leaf, and $n$ is the number of the leaves.

$$
\varepsilon(t)=\left[\varepsilon_{1}(t), \varepsilon_{2}(t), \varepsilon_{3}(t), \cdots, \varepsilon_{n}(t)\right]^{T} \in R^{n} \quad \text { is } \quad \text { the }
$$

Synchronized error vector, as shown in Eq. 5 .

$$
\varepsilon=T e
$$

where, $e=\left[e_{1}, e_{2}, \cdots, e_{n}\right]^{T}, T$ is the Synchronized transfer matrix,described as Equation.6.

$$
T=\left[\begin{array}{ccccc}
I & -I & & & \\
& I & -I & & \\
& & \ddots & & \\
& & & I & -I \\
I & & & & I
\end{array}\right]
$$

Obviously, if the Synchronized error $\varepsilon_{i}=0$, for all $i$ ( $i=$ $1,2, \ldots, n)$, the Synchronized goal Eq. 30 can be achieved. The Synchronized error represents the degree of coordination amongst the actuated leaves in the formation, and is not equivalent to the conventional tracking error. Employment of the synchronized error provides each leaf with motion information both from itself and from the other leaves. Hence, the motions of all leaves are well coordinated. The crosscoupled control schematic diagram is shown in Figure 2.

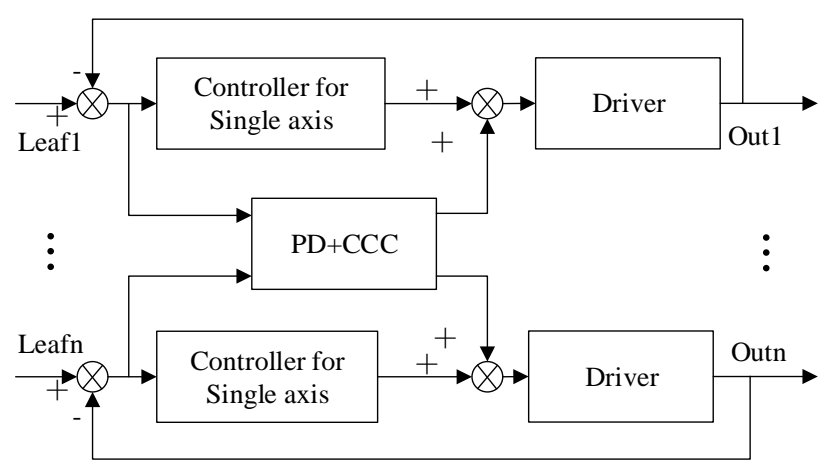

Figure 2. Schematic diagram of the structure of the crosscoupled controller

\subsubsection{Iterative learning control}

In 1978, Japanese scholars Mihara and Uchiyama published a research paper on Iterative Learning Control [19]. However, due to the publication in Japanese, the paper did not attract much international attention on the theoretical method. It was not until 1984 that the exact concept proposed by Arimoto, a Japanese scholar, for the iterative learning control method was also presented and a very comprehensive exposition and analysis of the iterative learning control method was carried out in this research paper. In the paper, the expression of Arimoto-type iterative learning control is given. Since then, iterative learning control has attracted more and more scholars' extensive research [20].

The classical Arimoto-type iterative learning control law is shown in Eq. 7:

$$
u_{i+1}=u_{i}(t)+L e_{i}(t)
$$

where, $u_{i+1}$ represents the control input for the $(i+1) t h$ iteration, $u_{i}$ represents the control input for the $i t h$ iteration, $e_{i}$ is the error of the ith iteration. If the desired trajectory is $r$ then $e_{i}=r-u_{i}, L$ means learning law. The above control law is a continuous iterative learning control law whose purpose is to keep $\lim _{i \rightarrow \infty} e_{i}=0$ by iteratively learning the control input of system. Iterative learning algorithm block diagram shown in Figure 3.

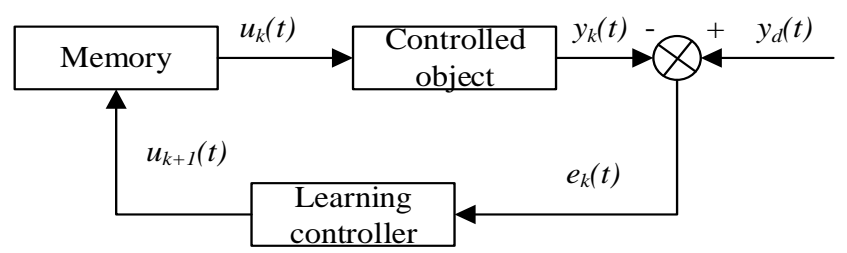

Figure 3. Iterative learning control algorithm diagram

On this basis, more and more scholars discussed the applicability of iterative learning control, and analyzed the iterative learning laws of $\mathrm{P}, \mathrm{D}$ and PID respectively. PID iterative learning control law described as:

$u_{i+1}(k)=u_{i}(k)+K_{\mathrm{p}} e_{i}+K_{\mathrm{I}} \sum_{m=1}^{k+1} e_{i}(m)+K_{\mathrm{D}}\left(e_{i}(k+1)-e_{i}(k)\right) k \in(0, N-1)$

where, $K_{p}, K_{I}, K_{D}$ are three adjustable parameters, $N$ is the system's learning cycle, other variables are as described in Eq.7, $L$ can be described as:

$$
L \dot{e}_{i}(t)=K_{\mathrm{p}} e_{i}+K_{\mathrm{I}} \sum_{m=1}^{k+1} e_{i}(m)+K_{\mathrm{D}}\left(e_{i}(k+1)-e_{i}(k)\right) k \in(0, N-1)
$$

\subsubsection{Iterative learning control based on cross-coupled}

Iterative learning control strategy based on Cross-coupled can be described as:

$$
u_{i+1}=u_{i}(t)+L_{e} e_{i}(t)+C L_{\varepsilon} \varepsilon_{i}(t)
$$

where, $u_{i+1}$ is control input, $L_{e}$ is the learning law of ILC, $L_{\varepsilon}$ is control law of cross-coupled, $C$ is the synchronized coefficient (also called coupled operator), $e_{i}(t)$ and $\varepsilon_{i}(t)$ are the leave tracking error and synchronized error, respectively. PD CCC and PD ILC are used in this study, and described as shown in Eq. 11:

$$
\left\{\begin{array}{l}
L_{e} e_{i}(t)=K_{\mathrm{P}}^{\mathrm{ILC}} e_{i}(t)+\sum_{m=1}^{t+1} K_{\mathrm{D}}^{\mathrm{ILC}}\left(e_{i}(t+1)-e_{i}(t)\right) \\
L_{\varepsilon} \varepsilon_{i}(t)=K_{\mathrm{P}}^{\mathrm{CCC}} \varepsilon_{i}(t)+K_{\mathrm{D}}^{\mathrm{CCC}}\left(\varepsilon_{i}(t+1)-e_{i}(t)\right)
\end{array}\right.
$$


where, $K_{P}^{\mathrm{ILC}}, K_{I}^{\mathrm{ILC}}$, and $K_{D}^{\mathrm{ILC}}$ are the proportional coefficient, integral coefficient and differential coefficient of PID iterative learning control, $K_{P}^{\mathrm{CCC}}$ and $K_{D}^{\mathrm{CCC}}$ are proportional coefficient and differential coefficient of the PD crosscoupled control, respectively.

\section{EXPERIMENTAL AND RESULTS}

\subsection{Experimental platform and equipment}

The experimental apparatus includes the leaves of multileaf collimator shown in Figure 4, the drive unit of MLC shown in Figure 5, and a control circuit board as shown in Figure 6. The TMS320F812 is taken as the control core, and the power drive module is composed of single-phase diode bridge rectifier, large capacitance filter and three-phase twolevel IGBT inverter. The incremental photoelectric encoder is used to detect the speed and position of the motor that drive the leaves.

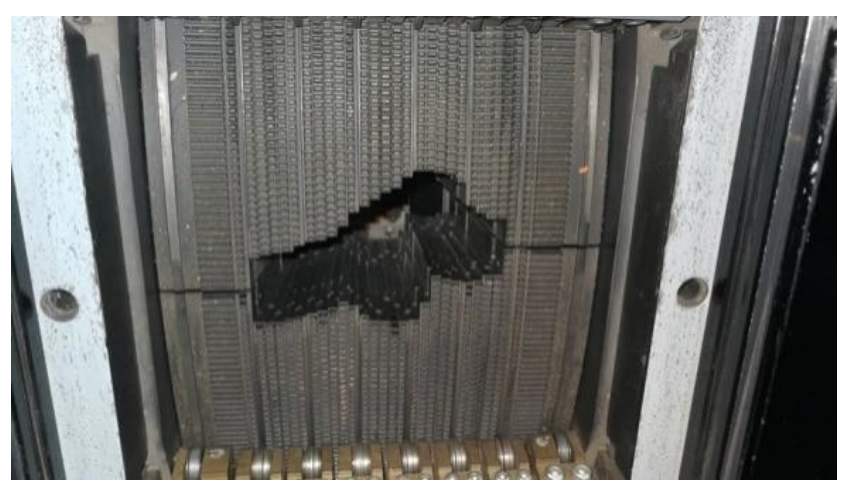

Figure 4. Leaves of multi -leaf collimator

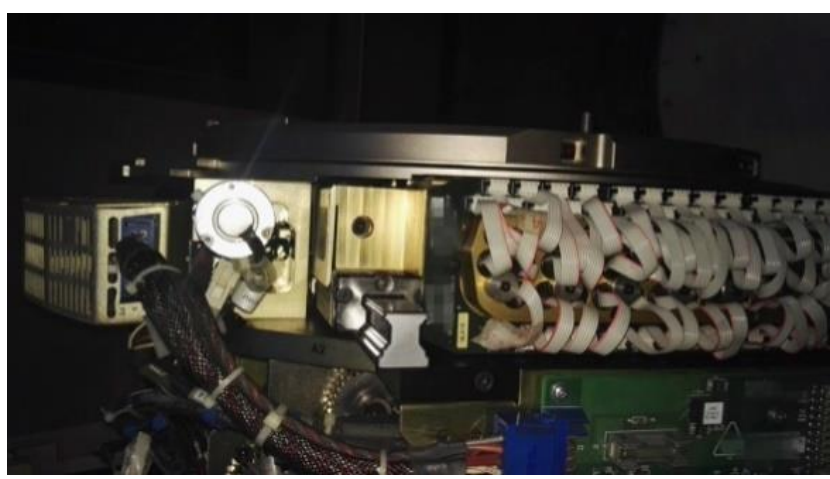

Figure 5. Drive unit of multi-leaf collimator

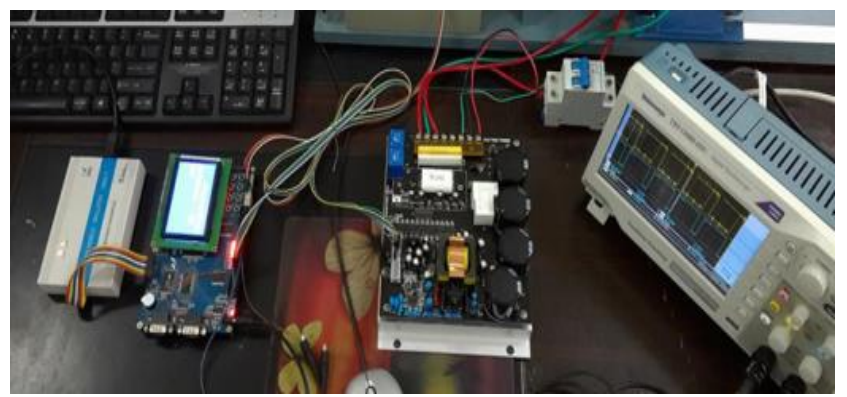

Figure 6. Control circuit board

\subsection{Results and analysis}

The validity of the proposed method can be verified by arbitrarily selecting three adjacent leaves as the controlled object.

First, an independent PD ILC control strategy to achieve multi-leaf synchronized control, then the iterative learning synchronized control strategy based on cross-coupling control is verified to show the superiority of the proposed method.

3.2.1 Results of PD ILC controller for MLC leaves based on cross-coupled

In the Equation.1, $M=\operatorname{diag}(3+0.5 * q(i)), \quad C=$ $\operatorname{diag}(3+0.3 * q(i) * 0.5 * \sin (\dot{q}(i))), \quad \alpha=0.3$. where, $q(i)$ is the input of the ith leaf. The input of the three leaves are $q_{1} d=\sin (3 t), \quad q_{2} d=\cos (3 t), \quad q_{3} d=2 \sin (2 t)$ respectively. The parameters of PD ILC are $K_{p}=$ [50 00 0; 050 0; 0 0 50] and $K_{d}=$ [50 00 0; $\left.0 \begin{array}{lllll}50 & 0 ; 0 & 0 & 50\end{array}\right]$. The position tracking curves of leaves are shown in the Figure 7, the motor torque outputs are shown in the Figure 8, the position errors of leaves are shown in the Figure 9.
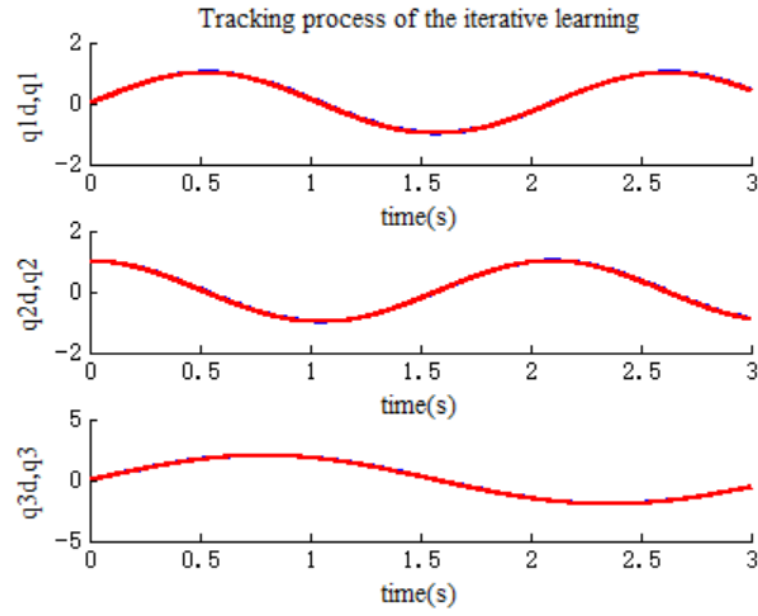

Figure 7. Tracking process curve of leaf position based on iterative learning
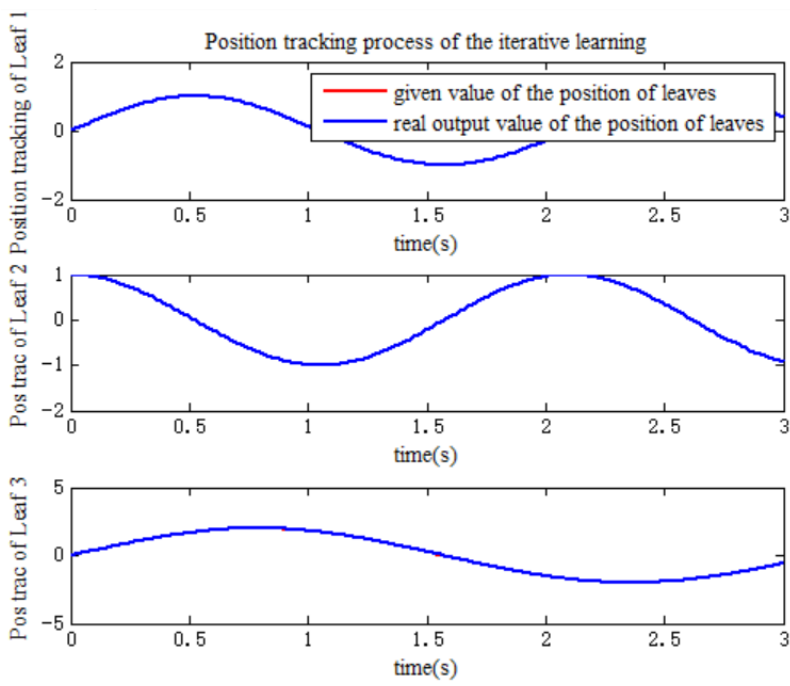

Figure 8. Tracking results curve of leaf position based on iterative learning 


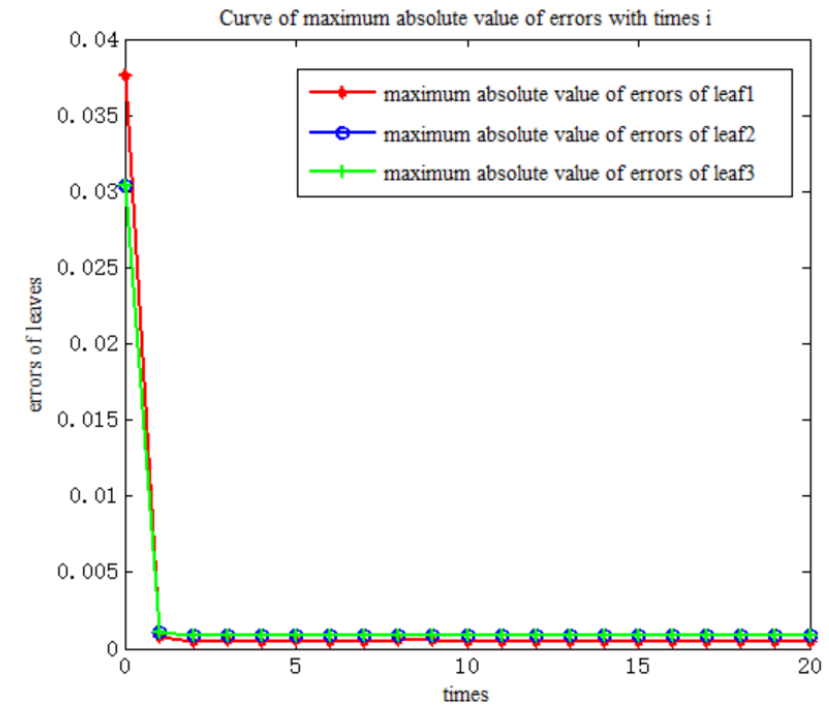

Figure 9. Curve of maximum absolute value of errors based on iteration learning

3.2.2 Results of iterative learning control based on crosscoupled
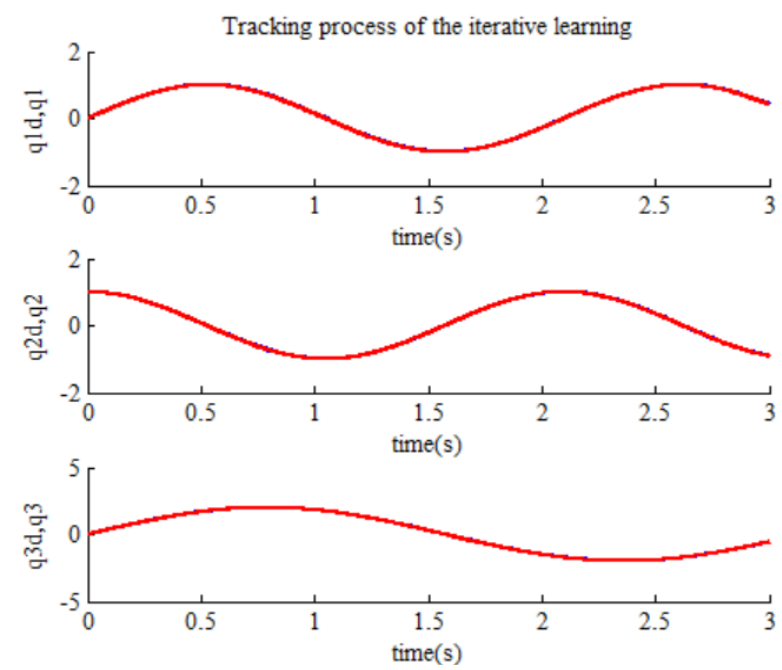

Figure 10. Tracking process curve of leaf position based on proposed method
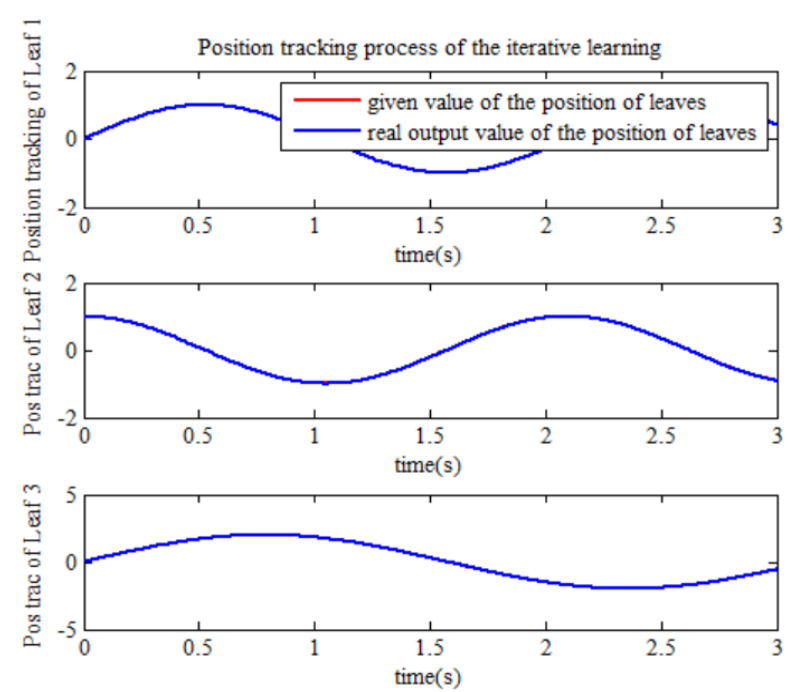

Figure 11. Tracking results curve of leaf position based on proposed method

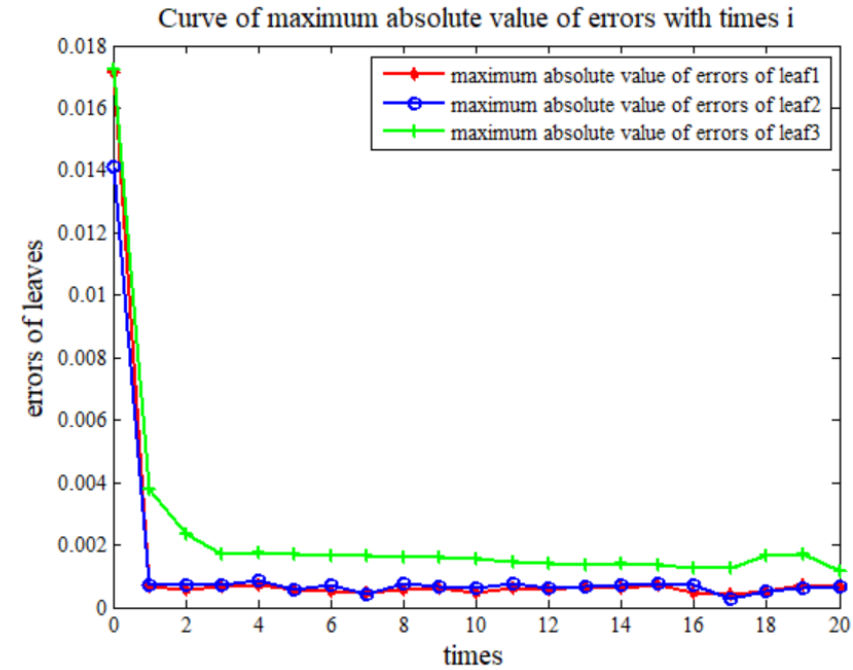

Figure 12. Curve of maximum absolute value of errors based on proposed method

The parameters of the proposed method in this study is the same as that of the independent PD ILC controller are $K_{p}^{\mathrm{ILC}}=$

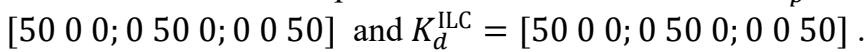
The other parameters of $\mathrm{PD}$ CCC are $K_{p}^{\mathrm{CCC}}=$ [50 00 0; 0500 0; 0 0 50] and $K_{d}^{\mathrm{CCC}}=\left[\begin{array}{lllllll}50 & 0 & 0 ; 0 & 50 & 0 ; 0 & 0 & 50\end{array}\right]$. The input of the three leaves are also $q_{1} d=\sin (3 t), q_{2} d=$ $\cos (3 t), q_{3} d=2 \sin (2 t)$ The results of the proposed method in this paper are as follows: the position tracking curves of leaves are shown in Figure 10, motor torque outputs are shown in Figure 11, position errors of leaves are shown in Figure 12.

From the experimental results, it can be shown that the leaf trajectory of MLC can track the trajectory that is outputted by the treatment planning system to satisfy the requirements of dynamic IMRT. Taking leaf 1 as the research object, the starting value of the maximum absolute value of errors using independent ILC control strategy is 0.0376 , the starting value of the maximum absolute value of errors using the method proposed in this study is 0.0171 , that smaller than the results obtained with the independent ILC method, the synchronized position error of the leaf can be reduced from the beginning.

The control strategy proposed in this study for dynamic IMRT is effective and feasible.

\section{CONCLUSIONS}

To ensure the consistency between MLC leave position and the position pre-set in the radiotherapy plan, this paper designs the iterative learning synchronized control strategy according to the cross-coupled control theory and the features of synchronize control of multi-leaf of MLC.

To eliminate the effect of position error between leaves on radiotherapy, the synchronized position error between adjacent leaves was defined and taken as the input for the synchronized control strategy. Then, the iterative learning synchronized control strategy was put forward based on cross-coupled control, aiming to eliminate the synchronized position error between leaves and satisfy the IMRT requirements. This strategy ensures that MLC leaves move along the planned trajectory to achieve the purpose of dynamic IMRT. 


\section{ACKNOWLEDGMENT}

The Program for Changjiang Scholars and Innovative Research Team in University (IRT 16R36) and Foundation of a Hundred Youth Talents Training Program of Lanzhou Jiaotong University.

\section{REFERENCES}

[1] Datta, N.R., Ordóñez, G.S., Gaipl, U.S., Paulides, M.M., Crezee, H., Gellermann, J., Marder, D., Puric, E., Bodis, S. (2015). Local hyperthermia combined with radiotherapy and-/or chemotherapy: Recent advances and promises for the future. Cancer Treatment Reviews, 41(9):

742-753.

https://doi.org/10.1016/j.ctrv.2015.05.009

[2] Poulsen, P.R., Cho, B., Ruan, D. (2010). Dynamic multi-leaf collimator tracking of respiratory target motion based on a single kilovoltage imager during arc radiotherapy. International Journal of Radiation Oncology Biology Physics, 77(2): 600-607. https://doi.org/10.1016/j.ijrobp.2009.08.030

[3] Arumugam, S., Xing, A., Goozee, G., Holloway, L. (2014). Independent calculation-based verification of IMRT plans using a 3D dose-calculation engine. Medical Dosimetry, 38(4): 376-384. https://doi.org/10.1016/j.meddos.2013.04.005

[4] Zaferullah, Z., Mishra, B.K., Bansode, R., Vidwans, M., Dsouza, K. (2014). Control of collimator for conformal radiation therapy based on FPGA implementation. Global Journal of Research in Engineering, 14(4): 27-31.

[5] Xin, Z. (2013). Parameter optimization of PID controller based on PSO for multi-leaf collimator. Indonesian Journal of Electrical Engineering and Computer Science, 11(10): 6127-6134 https://doi.org/10.3969/j.issn.1001-4373.2013.06.004

[6] Kothare, M.V., Campo, P.J., Morari, M., Nett, C.N. (1994). A unified framework for the study of antiwindup designs. Automatica, 30(12): 1869-1883. https://doi.org/10.1016/0005-1098(94)90048-5

[7] Zhang, X., Li, J.X., Dang, J.W., Wang, Y.P. (2017). Design and parameters optimization of the fractional order anti-windup controller for multileaf collimator. Journal of Engineering Science and Technology Review, 10(2): $\quad 35-41 . \quad$ https://doi.org/10.2991/icsnce16.2016 .112

[8] Hou, J.H. (2006). Study on key techniques of multi leaf collimator radiotherapy system. PhD Thesis. Dalian: Dalian University of Technology.

[9] Cavanini, L., Colombo, L.M., Ippoliti, G., Orlando, G. (2017). Development and experimental validation of a LQG control for a pre-compensated multi-axis piezo system. Industrial Electronics (ISIE), IEEE 26th International Symposium on. IEEE, pp. 460-465. https://doi.org/ 10.1109/ISIE.2017.8001290
[10] Koren Y. (1980). Cross-coupled biaxial computer control for manufacturing systems. Journal of Dynamic Systems, Measurement, and Control, (102): 265-271. https://doi.org/10.1115/1.3149612

[11] Sun, D., Wang, C. (2007). Controlling swarms of mobile robots for switching between formations using synchronization concept. IEEE International Conference on Robotics and Automation, pp. 23002305. https://doi.org/10.1109/ROBOT.2007.363662

[12] Lan, T. (2010). Research on synchronized control of multi-fingered anthropopathy dexterous robot hand. $\mathrm{PhD}$ Thesis. Harbin: Harbin Institute of Technology.

[13] Zhong, G., Shao, Z., Deng, H., Ren, J.L. (2017). Precise position synchronous control for multi-axis servo systems. IEEE Transactions on Industrial Electronics, 64(5):

$3707-3717$ https://doi.org/10.1109/TIE.2017.2652343

[14] Bristow, D.A., Tharayil, M., Alleyne, A.G. (2006). A survey of iterative learning control. IEEE Control Systems, 26(3): 96-114. https://doi.org/10.1109/mcs.2006.1636313

[15] Sampson, P., Freeman, C., Coote, S., Demain, S., Feys, P., Meadmore, K., Hughes, A.M. (2016). Using functional electrical stimulation mediated by iterative learning control and robotics to improve arm movement for people with multiple sclerosis. IEEE Transactions on Neural Systems and Rehabilitation Engineering, 24(2): $235-248$ https://doi.org/10.1109/TNSRE.2015.2413906

[16] Page, R.F., Abbott, N.L., Davies, J., Dyke, E.L., Randles, H.J., Velthuis, J.J., Fletcher, S., Gregory, S.D., Hall, C., John, A., Lawrence, H., Stevens, P.H., Hugtenburg, R.P., Tunbridge, V. (2014). Using a monolithic active pixel sensor for monitoring multi-leaf collimator positions in intensity modulated radiotherapy. IEEE Transactions on Nuclear Science, 61(1): 71-74. https://doi.org/10.1109/TNS.2013.2287609

[17] $\mathrm{Xu}, \mathrm{W}$. (2009). Study on multi-axis motion control system based on real-time ethernet. Wuhan: Huazhong University of Science and Technology. https://doi.org/10.7666/d.d088496

[18] Ling, J., Ming, M., Feng, Z., Xiao, X.H. (2017). A Master-Slave Cross-Coupled Iterative Learning Control for repetitive tracking of nonlinear contours in multiaxis precision motion systems. Acta Automatica Sinica, 43(12): $2127-2140$ https://doi.org/10.16383/j.aas.2017.c160725

[19] Uchiyama, M. (1978). Determination of malonaldehyde precursor in tissues by thiobarbituric acid test. Analytical Biochemistry, 86(1): 271-278. https://doi.org/ 10.1016/0003-2697(78)90342-1

[20] Arimoto, S., Kawamura, S., Miyazaki, F. (1984). Bettering operation of robots by learning. Journal of Robotic Systems, 1(2): 123-140. https://doi.org/10.1002/rob.4620010203 